\title{
Thinking pain away
}

The brain controls many processes, including sensory perception and voluntary action, without the need for conscious thought. An ability to purposefully control the activation of particular mechanisms in distinct brain areas could have far-reaching implications for the treatment of disease, and a group of US researchers studying pain have now shown that localized control can be exerted in at least one brain area, with significant effect.

Chronic pain is a widespread and costly medical problem, and many patients never attain adequate relief. Research suggests that the rostral anterior cingulate cortex (rACC) is involved in the perception and

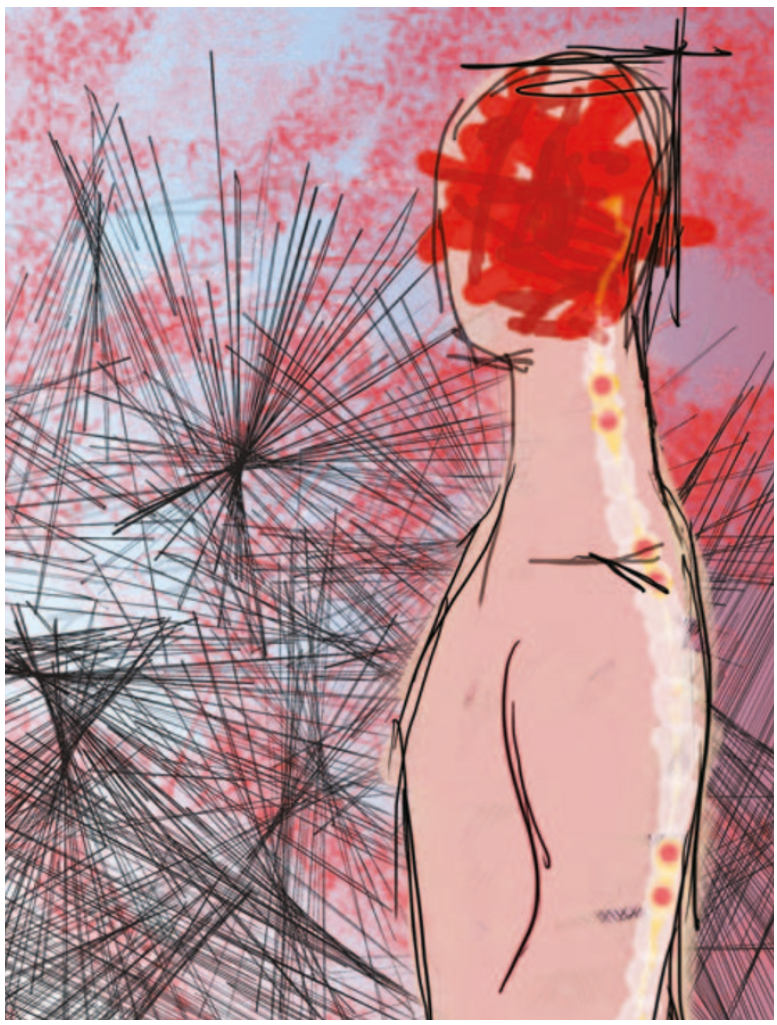

regulation of pain, and deCharms and co-workers set out to investigate whether control over its activation could be used to vary perceptions of pain.

Their study evaluated both healthy volunteers and patients with chronic pain, and real-time functional MRI (rtfMRI) was used to provide information about rACC activation as it occurred. Participants were shown line chart and video images to represent rtfMRI measurements of their levels of rACC activation. Through the feedback provided by these images, the healthy participants were trained to increase and then decrease their rACC activation over two consecutive minutes, with a $30 \mathrm{~s}$ application of a noxious heat stimulus beginning $10 \mathrm{~s}$ into each minute. Patients with chronic pain were also trained to increase and decrease their rACC activity, but were not subjected to the external stimulus.

After training, healthy participants reported increased pain perception during test periods in which they were controlling increases in rACC activation compared with when they controlled decreases.
Control groups that had undergone various similar training procedures without the images representing their $\mathrm{ACC}$ activation reported significantly less control over pain perception and smaller variations in pain intensity.

The patients with chronic pain all reported a considerable decrease in baseline pain levels after the training procedure, in some cases of more than $50 \%$, whereas a control patient group, in which autonomic rather than rACC activation feedback had been given, experienced much smaller decreases in pain.

Controlling pain through realtime imaging may be a clinical possibility for the future. Whether similar control can be exerted over other brain areas, and what other applications this technique might have remain to be discovered.

\section{Sarah Archibald}

ORIGINAL RESEARCH PAPER deCharms, R. C. et al. Control over brain activation and pain learned by using real-time functional MRI. Proc Natl Acad. Sci. USA 102, 18626-18631 (2005) FURTHER READING Pain collection. Nature Rev. Neurosci. 6 (Suppl.), 1-54 (2005)| Salomons, T. V.et al. Perceived controllability modulates the neural response to pain. J. Neurosci. 24, 7199-7203 (2004) 